\title{
Oblate deformation of light neutron-rich even-even nuclei
}

\author{
Ikuko Hamamoto ${ }^{1,2}$ \\ 1 Riken Nishina Center, Wako, Saitama 351-0198, Japan \\ 2 Division of Mathematical Physics, \\ Lund Institute of Technology at the University of Lund, Lund, Sweden
}

\begin{abstract}
Light neutron-rich even-even nuclei, of which the ground state is oblately deformed, are looked for, examining the Nilsson diagram based on realistic Woods-Saxon potentials. One-particle energies of the Nilsson diagram are calculated by solving the coupled differential equations obtained from the Schrödinger equation in coordinate space with the proper asymptotic behavior for $r \rightarrow \infty$ for both one-particle bound and resonant levels. The eigenphase formalism is used in the calculation of one-particle resonant energies. Large energy gaps on the oblate side of the Nilsson diagrams are found to be related to the magic numbers for the oblate deformation of the harmonic-oscillator potential where the frequency ratios $\left(\omega_{\perp}: \omega_{z}\right)$ are simple rational numbers. In contrast, for the prolate deformation the magic numbers obtained from simple rational ratios of $\left(\omega_{\perp}: \omega_{z}\right)$ of the harmonic-oscillator potential are hardly related to the particle numbers, at which large energy gaps appear in the Nilsson diagrams based on realistic Woods-Saxon potentials. The argument for an oblate shape of ${ }_{14}^{42} \mathrm{Si}_{28}$ is given. Among light nuclei the nucleus ${ }_{6}^{20} \mathrm{C}_{14}$ is found to be a good candidate for having the oblate ground state. In the region of the mass number $A \approx 70$ the oblate ground state may be found in the nuclei around ${ }_{28}^{76} \mathrm{Ni}_{48}$ in addition to ${ }_{28}^{64} \mathrm{Ni}_{36}$.

PACS numbers: 21.60.Ev, 21.10.Pc, 27.30.+t, 27.40.+z, 27.50.+e
\end{abstract}




\section{INTRODUCTION}

The ground states of some nuclei are described by densities and mean fields that are spherical, while others are deformed. Quadrupole deformation is by far the most important type of deformation, and the features of most deformed nuclei are consistent with an axially symmetric shape. Prolate shape is absolutely dominant in axially-symmetric quadrupoledeformed nuclei observed so far, while in the absence of pair correlation one obtains the number of prolate systems equal to that of oblate ones in the simple models such as harmonicoscillator and single-j-shell. A simple intuitive reason for the absolute dominance of prolate shape in observed deformed nuclei is not yet clarified [1]. Thus, at present one may say that the shell-structure which strongly favors oblate shape is needed for the realization of oblate nuclei.

Recently the neutron-rich nucleus ${ }_{14}^{42} \mathrm{Si}_{28}$ is reported to show a rotational spectrum [2], namely the ratio of observed excitation energies is $E\left(4^{+}\right) / E\left(2^{+}\right)=2.93(5)$ where $E\left(I^{\pi}\right)$ expresses the excitation energy of the lowest level with the spin-parity $I^{\pi}$, though the spinassignment of the $4^{+}$state is not yet pinned down experimentally. The observed value of $\mathrm{E}\left(2^{+}\right)$is not small enough, namely the relatively low moment of inertia indicates an oblate deformation though a decisive experimental evidence for the oblate shape is not yet obtained. One may wonder the reason why the nucleus with the neutron-number $N=28$ is deformed and not spherical, as the particle number 28 is a well-known magic-number in the $\mathrm{j}$-j coupling shell-model [3, 4]].

When I look for even-even nuclei in the range of $6 \leq Z \leq 40$, of which the observed electric quadrupole moment of the first-excited $2^{+}$state is clearly positive corresponding to an oblate shape (or a fluctuation towords oblate shape), I find [5, 6] only the following five nuclei: ${ }_{6}^{12} \mathrm{C}_{6},{ }_{14}^{28} \mathrm{Si}_{14},{ }_{16}^{34} \mathrm{~S}_{18},{ }_{18}^{36} \mathrm{Ar}_{18}$ and ${ }_{28}^{64} \mathrm{Ni}_{36}$. In addition, the ground state of the proton-rich $N=Z$ nucleus ${ }_{36}^{72} \mathrm{Kr}_{36}$ seems to have most likely an oblate shape [7].

Examining the values of proton- and neutron-numbers of the oblate nuclei mentioned above reminds me of the magic numbers of oblate deformations with the frequency ratio $\left(\omega_{\perp}\right.$ : $\left.\omega_{3}\right)=(1: 2)$ and (2:3) in the deformed harmonic-oscillator (h.o.) potential (see Figure 6-48 of [8]). Namely, those magic numbers are $Z=N=6,14,26,44,68, \ldots$ for the $(1: 2)$ deformation and $N=Z=6,8,14,18,28,34,48,58, \ldots$ for the $(2: 3)$ deformation. When the neutronand/or proton-numbers are equal to one of those magic numbers, the system in the deformed 
h.o. potential is supposed to be especially stable for respective $\left(\omega_{\perp}: \omega_{z}\right)$ deformations, though the total deformation is determined by both proton- and neutron-numbers. In the deformed h.o. model those magic numbers and the degeneracy of one-particle levels in the shells above and below can be understood in terms of a simple intuitive picture [8]. In reality, besides the difference between the h.o. potential and realistic potentials, one should take into account that the neutron shell-structure around the Fermi level may drastically change in nuclei towards neutron drip line [9-11].

In the present paper the possible presence of the oblate shape of the ground state of lighter neutron-rich even-even nuclei is explored, studying the shell-structure of the Nilsson diagram (namely neutron one-particle spectra as a function of axially-symmetric quadrupole deformation) based on realistic deformed Woods-Saxon potentials. The deviation from (the similarity to) the magic numbers of the deformed h.o. potential is discussed for prolate (oblate) shape.

In Sec. II the brief summary of the model used is given, while numerical results, the simple interpretation and the direct consequences are presented in Sec. III. Conclusions and discussions are given in Sec. IV.

\section{MODEL}

The basic points of the model used in the present paper are very similar to those of Ref. [11]. While weakly-bound neutrons in neutron-rich nuclei close to the neutron drip line make a contribution especially to the tail of the self-consistent nuclear potentials, the major part of the nuclear potential for neutrons is provided by protons, which are deeply bound in the case of neutron-rich nuclei. And, since the effective interaction to be used in the Hartree-Fock calculations of neutron-rich nuclei far away from the stability line is not yet well pinned down, in the present study I use Woods-Saxon potentials with the standard parameters which are described on p.239 of Ref. [12].

The calculation of energies of bound particles is an eigenvalue problem solving the coupled differential equations derived from the Schrödinger equation in coordinate space, together with the correct asymptotic behavior of bound wave-functions in respective channels for $r \rightarrow \infty$. In contast, one-particle resonant energies are obtained by solving the coupled differential equations in coordinate space using the asymptotic behavior of scattering states 
in respective channels and are defined as the energies, at which one of the eigenphases increases through $\pi / 2$ as the energy increases [13, 14].

One-particle resonance is absent if none of the eigenphases increase through $\pi / 2$ as the energy increases. As the energy increases, the width of a given resonant level becomes generally larger, and finally at a certain energy the one-particle resonant level with a given $\Omega^{\pi}$ (or $\ell j$ in spherical cases) is no longer obtained, where $\Omega$ expresses the angular-momentum component of the particle along the symmetry axis. The disappearance of those one-particle resonant levels can occur just above the Fermi level of some neutron drip line nuclei. See, for example, the Nilsson diagram for neutrons of ${ }_{6}^{20} \mathrm{C}_{14}$ shown in Fig. 2. For simplicity, the calculated widths of one-particle resonant levels are not always given, because the widths are not of major interest in the present work. When the energy gap in one-particle spectra of nuclei close to the neutron drip line is dicussed, it is important to explore the shell structure including one-particle resonant levels.

I use the knowledge that a large energy gap in one-particle spectra for some deformation in the Nilsson diagram leads to the stability of the system for the deformation and the particlenumber. The deformation parameter $\beta$ of the ground state obtained from experimental data is relatively large for lighter nuclei, but certainly smaller than $|\beta|<0.6$. Therefore, in the following I study the deformation region of $|\beta|<0.6$.

\section{NUMERICAL RESULTS}

It is known that for light stable nuclei the realistic Nilsson diagrams for protons and neutrons are not so different. The Nilsson diagram for well-bound protons has been studied in various publications, and even the diagrams based on modified-oscillator potentials can be safely used to find out the dependence of the shell structure on the proton number and deformations. Whether a given nucleus is deformed or not depends on both proton- and neutron-numbers, and the oblate ground-state of even-even nuclei is rather rare. Thus, when possible lighter neutron-rich oblate nuclei are searched for, the proton numbers are fixed to be those that are known to be favored by oblate shape.

The volume conservation is taken into account in Nilsson diagrams shown in the present paper, while it is neglected in those of previous publications [10, 11]. The neglect hardly affects the discussion of the shell-structure change in neutron-rich nuclei. 


\section{A. Oblate shape of the nucleus ${ }_{14}^{42} \mathbf{S i}_{28}$}

It is known that a large energy gap appears at the proton number $Z=14$ on the oblate side of the Nilsson diagram obtained from both the modified-oscillator potential and any realistic Woods-Saxon potential appropriate for stable nuclei. The particle number 14 is also the magic number for both the (1:2) and (2:3) oblate deformations in the h.o. potential.

In Fig. 1 the Nilsson diagram for neutrons of ${ }_{14}^{42} \mathrm{Si}_{28}$ is shown. The energy distance $\varepsilon\left(2 p_{3 / 2}\right)-\varepsilon\left(1 f_{7 / 2}\right)$ is only $1.65 \mathrm{MeV}$, which is much smaller than the $N=28$ energy gap known in the $\mathrm{j}$-j coupling shell-model. That means, the spherical shape is not particularly favored by ${ }^{42} \mathrm{Si}$. It is in fact known (for example, see Fig. 2 in Ref. [11]) that the $2 p_{3 / 2}$ and $1 f_{7 / 2}$ levels come very close to each other (or the level order can be even reversed), when those two levels become very weakly bound or low-energy resonant. In contrast, from both Fig. 1 of the present paper and Fig. 2 of Ref. [11] it is seen that a large energy gap is developed at $N=28$ on the oblate side. The gap corresponds exactly to the magic number 28 for the (2:3) oblate deformation of the h.o. potential shown in Fig. 6-48 of Ref. [8]. Namely, at $N=28$ on the oblate side of Fig. 1 four doubly-degenerate Nilsson levels with the h.o. principal quantum-number $N_{h o}=3$ are occupied, while none of $N_{h o}=2$ Nilsson orbits are occupied. (Note that one-particle levels of the h.o. model in Fig. 6-48 of Ref. [8] are classified by the quantum numbers, $\left(n_{\perp}, n_{z}\right)$, therefore, the one-particle levels are not always doubly-degenerate but have the degeneracy of $2\left(n_{\perp}+1\right)$.) In short, the possible oblate deformation of the nucleus ${ }^{42} \mathrm{Si}$ can be understood as a result of the combination of the facts: (i) the disappearance of spherical $N=28$ magic number due to the shell-structure change in very neutron-rich nuclei; (ii) the $N=28$ remains as "a magic number" for the moderate-size $(\beta \approx-0.4)$ oblate deformation in the same way as the magic number for the (2:3) deformation of the h.o. potential; (iii) the oblate shape is much favored by $Z=14$.

Other energy gaps of a considerable size found on the oblate side of Fig. 1 for $\beta \approx-0.4$ are $N=14$ and 18, which are also the magic numbers for the oblate $(2: 3)$ deformation of the h.o. potential. In contrast, it is noticed that the neutron numbers corresponding to the energy gaps of an appreciable size that are seen on the prolate side of Fig. 1 (for example, $N$ $=16,24$, and 28) do not correspond to the magic numbers for the prolate (3:2) deformation of the h.o. potential.

The different correspondence between the realistic Woods-Saxon potential and the h.o. 
potential for the prolate deformation from the oblate deformation seems to come mainly from the different behavior of the Nilsson one-particle levels connected to the high-j shell (the $1 f_{7 / 2}$ shell in the present case) on the prolate side from the oblate side. The different behavior of the high-j Nilsson levels was discussed in detail in Ref. [1] in relation to the numbers of oblate/prolate nuclei. On the oblate side the shell-structure coming from the unique presence of the high-j orbits in realistic potentials is disturbed soon after deformation sets in. In contrast, on the prolate side the shell-structure due to the large spin-obit splitting is kept in the range of the realistic quadrupole deformation and, thus, the particle number, at which a large energy gap occurrs, is considerably different from that of the h.o. potential.

\section{B. Possible oblate shape of the nucleus ${ }_{6}^{20} \mathbf{C}_{14}$}

One may try to find out good candidates for other light neutron-rich nuclei with an oblate shape. There is a large energy gap above the proton number $Z=6$ on the oblate side of the Nilsson diagram with realistic potentials as well as the deformed modified-oscillator potential. For example, see Fig. $5-1$ of Ref. [8]. Therefore, a promissing candidate is ${ }_{6}^{20} \mathrm{C}_{14}$. From the measured values, $E\left(2_{1}^{+}\right) \approx 1.6 \mathrm{MeV}[15,16]$ and $B\left(E 2 ; 2_{1}^{+} \rightarrow 0^{+}\right) \approx 7.5 e^{2} f m^{4}$ [16], the nucleus ${ }^{20} \mathrm{C}$ is most probably deformed. The occupation of the nearly degenerate $2 s_{1 / 2}$ and $1 d_{5 / 2}$ levels by several neutrons may well induce a deformation due to the nuclear Jahn-Teller effect [10, 11]. One question is whether or not the large energy gap at $N=14$ remains for the oblate deformation with $\beta \approx-0.4$, when those two single-particle levels, $2 s_{1 / 2}$ and $1 d_{5 / 2}$, become very weakly-bound. In Fig. 2 the Nilsson diagram for neutrons of ${ }^{20} \mathrm{C}$ is shown. The width of the $\Omega^{\pi}=1 / 2^{+}$level connected to the $2 s_{1 / 2}$ level becomes extremely large immediately above zero energy, and the level cannot survive as a resonant state for $\beta<-0.215$. In essence, there is no $\Omega^{\pi}=1 / 2^{+}$one-particle resonant level for $-0.6<\beta<-0.2$, of which the wave-function has an appreciable probability inside the nucleus, and, thus, a large energy gap appears at $N=14$. Therefore, the nucleus ${ }_{6}^{20} \mathrm{C}_{14}$ is a promising candidate for a light neutron-rich nucleus with an oblate shape.

In Fig. 2 large energy gaps on the oblate side are observed at $N=6,8$, and 14 . Those neutron numbers are the magic numbers for the (2:3) oblate deformation of the h.o. potential. The numbers of the orbits with a given $N_{h o}$ which lie below respective particle numbers are again exactly the same as those numbers for the (2:3) deformation. 


\section{Oblate neutron-rich nuclei in the $A \approx 70$ region}

In this slightly heavier mass region the one-particle level-density increases and the pair correlation may become more important, which is neglected in the present paper. Even in such cases, a low one-particle level-density at some deformation for a given particle-number in Nilsson diagrams usually plays a role in determining the deformation of the ground state of the system.

Examining the shell-structure around $\beta \approx-0.4$ in Fig. 3 of Ref. [11], large energy gaps are found at $N=36$ and 48 . The neutron numbers $N=34$ and 48 are the magic numbers for the (2:3) deformation of the h.o. potential. The $N=48$ gap in Fig. 3 of Ref. [11] corresponds exactly to the magic number 48 for the (2:3) deformation in the sense that five doubly-degenerate $N_{h o}=4$ levels are occupied while one doubly-degenerate $N_{h o}=3$ level is unoccupied. The occurrence of the large energy gap at $N=36$ instead of the magic number $N=34$ for the $(2: 3)$ deformation comes from the occupation of the lowest-lying doublydegenerate $\Omega^{\pi}=9 / 2^{+}$level, which originates from the $1 g_{9 / 2}$ level and is lowered steeply as oblate deformation increases. The wave function of the $\Omega^{\pi}=9 / 2^{+}$level is almost pure $1 g_{9 / 2}$ in the present range of $\beta$-values. The large energy gap at $N=36$ instead of $N=34$ comes from the presence of the high-j-shell orbit $\left(1 g_{9 / 2}\right.$ orbit) around the Fermi level in this neutron-number region, which is strongly pushed down by the large spin-orbit splitting that is absent in the h.o. model.

From the shell-structure in Fig. 3 of Ref. [11], one may note that possible candidates for the oblate ground-state of neutron-rich even-even nuclei in the $A \approx 70$ region are the nuclei around ${ }_{28}^{76} \mathrm{Ni}_{48}$ in addition to ${ }_{28}^{64} \mathrm{Ni}_{36}$.

As already pointed out in subsection III. A, it is seen from Fig. 3 of Ref. [11] that none of the large energy gaps for the prolate deformation with $\beta \approx 0.4$ correspond to the magic numbers for the $(3: 2)$ deformation of the h.o. potential, $N=,, 22,26,34,46,54, .$, .

\section{CONCLUSIONS AND DISCUSSIONS}

The possible oblate ground-state of light neutron-rich even-even nuclei is searched for, using the shell structure appearing in the Nilsson diagrams based on the Woods-Saxon potential with standard parameters. For nuclei close to the neutron-drip-line the shell- 
structure including one-particle resonant levels is studied.

It is shown that the neutron-numbers, at which large energy gaps appear for some oblate deformations of Nilsson diagrams based on the realistic potentials, can be easily related to the magic numbers coming from the degeneracy of the energy $\hbar\left(n_{\perp} \omega_{\perp}+n_{z} \omega_{z}\right)$ for the oblate deformation of the h.o. potential, in which the ratios of frequencies $\left(\omega_{\perp}: \omega_{z}\right)$ are simple rational numbers. Because of this simple property of the shell-structure on the oblate side of the Nilsson diagram, which is common to the Woods-Saxon potentials with realistic parameters, one may pretty reliably predict the light nuclei, of which the ground state may have an oblate shape. A good candidate for the oblate shape of very light nuclei is ${ }^{20} \mathrm{C}$. An oblate shape can be expected for ${ }_{14}^{42} \mathrm{Si}_{28}$, partly because $N=28$ is not really a magic number for spherical shape of the neutron-rich nucleus, instead, it is a magic number for the $(2: 3)$ oblate deformation of the h.o. potential and partly because $Z=14$ is a magic number for the $(2: 3)$ (and also (1:2)) oblate deformation. Indeed, one observes a large energy gap both at $N=28$ and $Z=14$ for the moderate-size (say, $\beta \approx-0.4$ ) deformation in the Nilsson diagram based on realistic Woods-Saxon potentials. In the mass-number $A \approx 70$ region the possible candidates for oblate shape are the nuclei around ${ }_{28}^{76} \mathrm{Ni}_{48}$ in addition to ${ }_{28}^{64} \mathrm{Ni}_{36}$.

In contrast, on the prolate side of the Nilsson diagram based on realistic Woods-Saxon potentials large energy gaps are hardly found at the particle numbers, which correspond to the magic numbers with a simple rational frequency-ratio $\left(\omega_{\perp}: \omega_{z}\right)$ of the h.o. potential. In other words, the particle numbers, at which large energy gaps appear on the oblate side of the Nilsson diagram, are quite common to various realistic potentials, while on the prolate side they may depend on some details of respective one-body potentials.

An oblate ground-state of ${ }^{42} \mathrm{Si}$ was also obtained in available shell-model calculations [17, 18] using various effective interactions with and without tensor forces. In those shellmodel calculations the h.o. wave-functions are always used and, therefore, the shell-structure change in neutron-rich nuclei due to the unique behavior of weakly-bound (and/or resonant) low- $\ell$ neutrons is absent. The shell-structure change may possibly be mimicked by adjusting some part of effective interactions together with input one-particle energies. On the other hand, the shape of the system is the property of the one-body mean field. It is the author's opinion that the explanation of the oblate shape of ${ }^{42} \mathrm{Si}$ given in the present paper is simple, intuitive and widely applicable.

The author is grateful to Professor K. Matsuyanagi for useful discussions which she had 
in the initial stage of the present work.

[1] I. Hamamoto and B. R. Mottelson, Phys. Rev. C 79, 034317 (2009), and references quoted therein.

[2] S. Takeuchi et al., Phys. Rev. Lett. 109, 182501 (2012).

[3] M. G. Mayer, Phys. Rev. 75, 1969 (1949).

[4] O. Haxel, J. H. D. Jensen and H. E. Suess, Phys. Rev. 75, 1766 (1949).

[5] P. Raghavan, At. Data Nucl. Data Tables 42, 189 (1989).

[6] N. J. Stone, At. Data Nucl. Data Tables 90, 75 (2005).

[7] A. Gade et al., Phys. Rev. Lett. 95, 022502 (2005); 96, 189901 (2006), Erratum.

[8] A. Bohr and B. R. Mottelson, Nuclear Structure (Benjamin, Reading, MA, 1975), Vol. II.

[9] A. Ozawa, T. Kobayashi, T. Suzuki, K. Yoshida, and I. Tanihata, Phys. Rev. Lett. 84, 5493 (2000).

[10] I. Hamamoto, Phys. Rev. C 76, 054319 (2007).

[11] I. Hamamoto, Phys. Rev. C 85, 064329 (2012).

[12] A. Bohr and B. R. Mottelson, Nuclear Structure (Benjamin, Reading, MA, 1969), Vol. I.

[13] R. G. Newton, Scattering Theory of Waves and Particles (McGraw-Hill, New York, 1966).

[14] I. Hamamoto, Phys. Rev. C 72, 024301 (2005); 73, 064308 (2006).

[15] M. Stanoiu et al., Phys. Rev. C 78, 034315 (2008).

[16] M. Petri et al., Phys. Rev. Lett. 107, 102501 (2011).

[17] F. Nowacki and A. Poves, Phys. Rev. C 79, 014310 (2009).

[18] Y. Utsuno, T. Otsuka, B. A. Brown, M. Honma, T. Mizusaki and N. Shimizu, Phys. Rev. C 86, 051301(R) (2012). 


\section{Figure captions}

Figure 1: Calculated one-particle energies for neutrons of ${ }_{14}^{42} \mathrm{Si}_{28}$ as a function of axiallysymmetric quadrupole deformation. Bound one-particle energies at $\beta=0$ are -8.50 , $-7.24,-2.42$, and $-0.77 \mathrm{MeV}$ for the $2 s_{1 / 2}, 1 d_{3 / 2}, 1 f_{7 / 2}$, and $2 p_{3 / 2}$ levels, respectively, while one-particle resonant $2 p_{1 / 2}$ and $1 f_{5 / 2}$ levels are obtained at $+0.20 \mathrm{MeV}$ with the width of $0.20 \mathrm{MeV}$ and $+3.25 \mathrm{MeV}$ with the width of $0.58 \mathrm{MeV}$, respectively, which are denoted by filled circles. The resonant $\Omega^{\pi}=1 / 2^{-}$level which is connected to the resonant $2 p_{1 / 2}$ level at $\beta=0$ cannot be obtained for $\beta<-0.19$ corresponding to $\varepsilon_{\Omega}>1.42 \mathrm{MeV}$. One-particle resonant energies for $\beta \neq 0$ are not plotted unless they are important for the present discussion. For simplicity, calculated widths of oneparticle resonant levels are not shown. The neutron numbers, 14, 18, 20 and 28, which are obtained by filling all lower-lying levels, are indicated with circles. One-particle levels with $\Omega=1 / 2,3 / 2,5 / 2$ and $7 / 2$ are expressed by solid, dotted, long-dashed and dot-dashed curves, respectively, for both positive and negative parities. The parity of levels can be seen from the $\ell$-values denoted at $\beta=0 ; \pi=(-1)^{\ell}$.

Figure 2: Calculated one-particle energies for neutrons of ${ }_{6}^{20} \mathrm{C}_{14}$ as a function of axiallysymmetric quadrupole deformation. Bound one-particle energies at $\beta=0$ are -7.66 , -1.28 and $-0.71 \mathrm{MeV}$ for the $1 p_{1 / 2}, 1 d_{5 / 2}$ and $2 s_{1 / 2}$ levels, respectively, while oneparticle resonant $1 d_{3 / 2}$ level is obtained at $+3.57 \mathrm{MeV}$ with the width of $3.02 \mathrm{MeV}$. The resonant $\Omega^{\pi}=1 / 2^{+}$level which is connected to the bound $2 s_{1 / 2}$ level at $\beta=0$ cannot be obtained for $\beta<-0.215$ corresponding to $\varepsilon_{\Omega}>0.38 \mathrm{MeV}$. On the other hand, the resonant $\Omega^{\pi}=1 / 2^{+}$level connected to the resonant $1 d_{3 / 2}$ level at $\beta=0$ cannot be obtained for $\beta>0.35$ corresponding to $\varepsilon<1.54 \mathrm{MeV}$, because in the deformation region the $s_{1 / 2}$ component becomes appreciable in the wave function. For smaller $\beta$ values the major component of the wave function of the $\Omega^{\pi}=1 / 2^{+}$level is $1 d_{3 / 2}$ and, thus, the resonant level survives. The neutron numbers, 6,8 and 14 , which are obtained by filling all lower-lying levels, are indicated with circles. One-particle levels with $\Omega=1 / 2,3 / 2$ and $5 / 2$ are expressed by solid, dotted and long-dashed curves, respectively, for both positive and negative parities. 


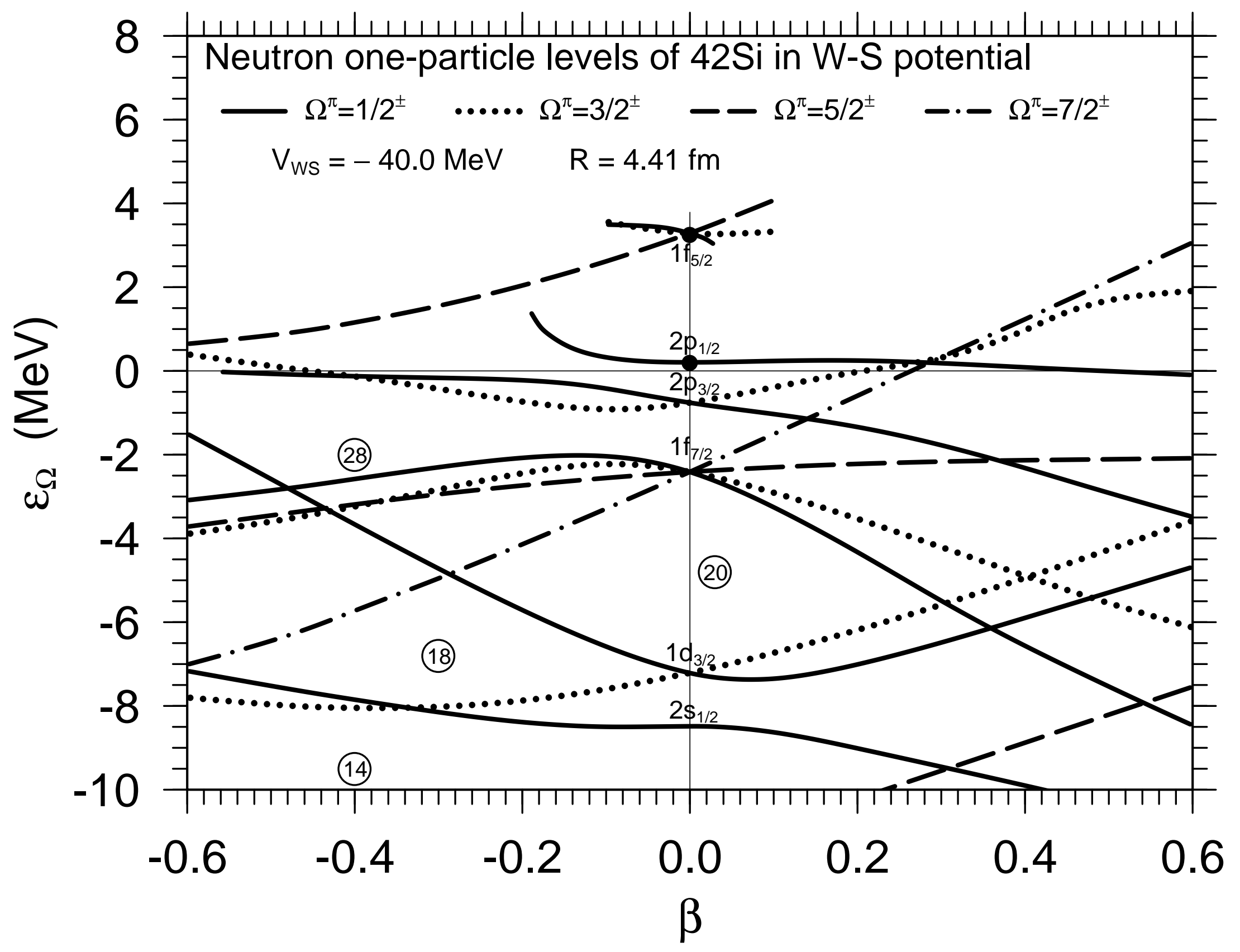




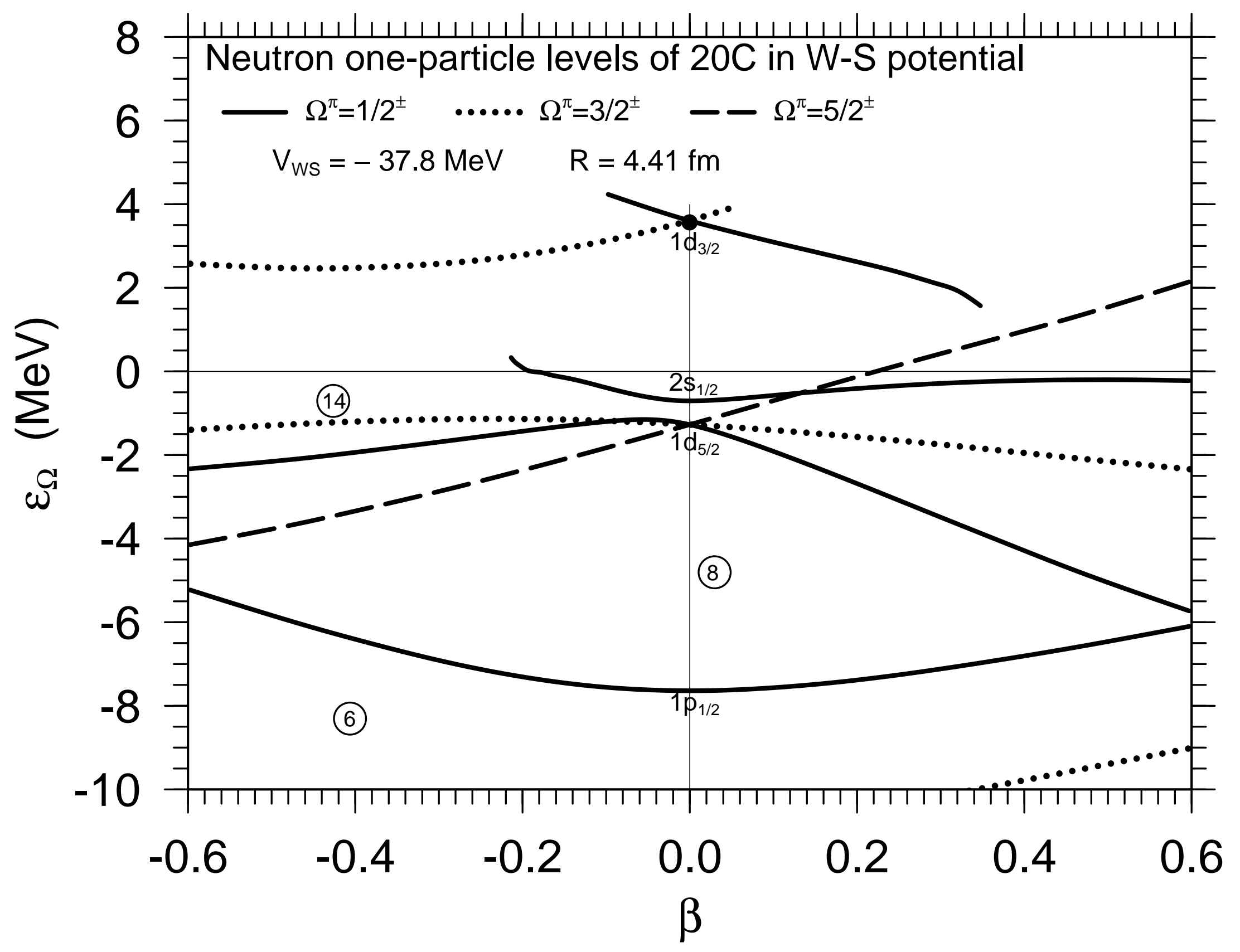

